Ferrata Storti Foundation

\title{
Role of interferon- $\gamma$ in immune-mediated graft failure after allogeneic hematopoietic stem cell transplantation
}

Pietro Merli, ${ }^{1}$ Ignazio Caruana, ${ }^{1}$ Rita De Vito, ${ }^{2}$ Luisa Strocchio, ${ }^{1}$ Gerrit Weber, ${ }^{1}$ Francesca Del Bufalo, ${ }^{1}$ Vanessa Buatois, ${ }^{3}$ Paolo Montanari, ${ }^{3}$ Maria Giuseppina Cefalo, ${ }^{1}$ Angela Pitisci, ${ }^{1}$ Mattia Algeri, ${ }^{1}$ Federica Galaverna,,${ }^{1}$ Concetta Quintarelli, ${ }^{1}$ Valentina Cirillo, ${ }^{1}$ Daria Pagliara, ${ }^{1}$ Walter Ferlin, ${ }^{3}$ Maria Ballabio, ${ }^{3}$ Cristina De Min $^{3}$ and Franco Locatelli ${ }^{1,4}$

Haematologica 2019

Volume 104(11):2314-2323

\section{Correspondence: \\ PIETRO MERLI \\ pietro.merli@opbg.net \\ Received: January 7, 2019. \\ Accepted: February 18, 2019. \\ Pre-published: February 21, 2019.}

doi:10.3324/haematol.2019.216101

Check the online version for the most updated information on this article, online supplements, and information on authorship \& disclosures: www.haematologica.org/content/104/11/2314

(C)2019 Ferrata Storti Foundation

Material published in Haematologica is covered by copyright. All rights are reserved to the Ferrata Storti Foundation. Use of published material is allowed under the following terms and conditions:

https://creativecommons.org/licenses/by-nc/4.0/legalcode. Copies of published material are allowed for personal or internal use. Sharing published material for non-commercial purposes is subject to the following conditions:

https://creativecommons.org/licenses/by-nc/4.0/legalcode, sect. 3. Reproducing and sharing published material for commercial purposes is not allowed without permission in writing from the publisher.
${ }^{1}$ Bambino Gesù Children's Hospital, Department of Pediatric Hematology/Oncology, Cellular and Gene Therapy, Rome, Italy; 'Bambino Gesù Children's Hospital, Department of Laboratories, Pathology Unit, Rome, Italy; ${ }^{3}$ Novimmune SA, Geneva, Switzerland and ${ }^{4}$ Department of Pediatrics, Sapienza, University of Rome, Rome, Italy

\section{ABSTRACT}

$\mathrm{P}$ athophysiology of graft failure (GF) occurring after allogeneic hematopoietic stem cell transplantation (HSCT) still remains elusive. We measured serum levels of several different cytokines/chemokines in 15 children experiencing GF, comparing their values with those of 15 controls who had sustained donor cell engraftment. Already at day +3 after transplantation, patients developing GF had serum levels of interferon (IFN)- $\gamma$ and CXCL9 (a chemokine specifically induced by IFN $\gamma$ ) significantly higher than those of controls $(8859 \pm 7502$ vs. $0 \mathrm{pg} / \mathrm{mL}, P=0.03$, and $1514.0 \pm 773$ vs. $233.6 \pm 50.1 \mathrm{pg} / \mathrm{mlL}, P=0.0006$, respectively). The role played by IFN $\gamma$ in HSCT-related GF was further supported by the observation that a rat anti-mouse IFN $\gamma$-neutralizing monoclonal antibody promotes donor cell engraftment in Ifngr $1^{-/}$mice receiving an allograft. In comparison to controls, analysis of bone marrow-infiltrating $\mathrm{T}$ lymphocytes in patients experiencing GF documented a predominance of effector memory $\mathrm{CD}^{+}$ cells, which showed markers of activation (overexpression of CD95 and downregulation of CD127) and exhaustion (CD57, CD279, CD223 and CD366). Finally, we obtained successful donor engraftment in 2 out of 3 children with primary hemophagocytic lymphohistiocytosis who, after experiencing GF, were re-transplanted from the same HLA-haploidentical donor under the compassionate use coverage of emapalumab, an anti-IFN $\gamma$ monoclonal antibody recently approved by the US Food and Drug Administration for treatment of patients with primary hemophagocytic lymphohistiocytosis. Altogether, these results suggest that the IFN $\gamma$ pathway plays a major role in GF occurring after HSCT. Increased serum levels of IFN $\gamma$ and CXCL9 represent potential biomarkers useful for early diagnosis of GF and provide the rationale for exploring the therapeutic/preventive role of targeted neutralization of IFN $\gamma$.

\section{Introduction}

Graft failure (GF), estimated to occur in 1-5\% of cases after myeloablative conditioning and in up to $30 \%$ of cases after reduced-intensity conditioning (RIC), ${ }^{1}$ still remains a relevant cause of morbidity and mortality after allogeneic hematopoietic stem cell transplantation (HSCT). ${ }^{2}$ Despite a slight reduction of its incidence over the last decade, mortality after GF remains as high as $11 \%{ }^{3}$ To date, in the absence of effective treatment options, re-transplantation, from either the same, or whenever possible, a different donor is considered the treatment of choice. ${ }^{2}$ Currently identified risk factors for GF include: i) human leukocyte antigen (HLA)-disparity and sex mismatch in the donor/recipient pair; ii) presence of 
donor-specific antibodies (DSA) in the recipient; iii) T-cell depletion (TCD) of the graft; iv) $\mathrm{ABO}$-blood group mismatch; v) use of RIC; vi) a diagnosis of non-malignant disorders (in particular thalassemia, severe aplastic anemia, SAA, and hemophagocytic lymphohistiocytosis, HLH); vii) viral infections; viii) low nucleated cell dose in the graft; and ix) the use of myelotoxic drugs in the posttransplant period. ${ }^{1-4}$

In the last two decades, several groups have investigated immune-mediated GF. In particular, it has been shown that immune-mediated GF is mainly caused by host $T$ and natural killer (NK) cells surviving the conditioning regimen, through a classical alloreactive immune response against non-shared, major (in case of HLA-partially-matched HSCT) or minor (in case of fully HLA-matched HSCT) histocompatibility antigens. ${ }^{2,5,6}$ However, to date the molecular pathways involved in immune-mediated GF have not yet been completely clarified. Indeed, since the inhibition of different pathways (including perforin-, FasL- ${ }^{-}$TNFR-1', and TRAIL-dependent cytotoxicity) did not prove to be efficient in preventing GF, the pathophysiological mechanisms responsible for GF seem to be multiple and likely to be redundant.' Nonetheless, consistently over the years, different groups have suggested a pivotal pathogenic role of IFN $\gamma$ in GF pathophysiology, ${ }^{8-14}$ through both direct [e.g. inhibition of hematopoietic stem cell (HSC) self-renewal, proliferative capacity, and multilineage differentiation $]^{10,11}$ and indirect (e.g. induction of FAS expression on HSC, with increased apoptosis in the presence of activated cytotoxic $\mathrm{T}$ cells $)^{8,12}$ effects.

Despite these experimental data, there has still not been any in vivo characterization of GF in humans. Indeed, although the expansion of host $\mathrm{CD}^{+} \mathrm{T}$ cells in patients experiencing GF has been previously demonstrated in vivo, ${ }^{15,16}$ a more detailed characterization of this cell population is lacking. Thus, we started a prospective study aimed at better characterizing the pathophysiology of GF, focusing on the identification of biological markers that: (i) could predict early the occurrence of GF in the clinical setting; and (ii) could be used as a therapeutic target with clinically available biological agents. For this purpose, we broadly investigated cytokine and chemokine levels in peripheral blood (PB), as well as the cellular features in bone marrow (BM) biopsies of patients experiencing this complication. After confirming in vivo a role of IFN $\gamma$-pathway in the development of GF, we also investigated in an animal model of GF whether the sole inhibition of IFN $\gamma$ would be able to prevent/treat GF. Finally, in view of these findings and the similarity between immune-mediated GF and HLH, we treated, in compassionate use (CU), with emapalumab, an anti-IFN $\gamma$ monoclonal antibody recently approved for the treatment of $\mathrm{HLH},{ }^{17}$ three patients with primary $\mathrm{HLH}$, who, after having experienced GF, underwent a second HSCT.

\section{Methods}

\section{Patients}

Patients aged from 0.3 to 21 years, who received an allograft from any type of donor/stem cell source between January $1^{\text {st }}$ 2016 and August 31 2017 at the IRCCS Bambino Gesù Children's Hospital in Rome, Italy, were considered eligible for the study. All patients or legal guardians provided written informed consent, and the entire research was conducted under institutional review board approved protocols and in accordance with the Declaration of Helsinki. The Bambino Gesù Children's Hospital Institutional Review Board approved the study.

\section{Cytokine profile}

In order to identify a cytokine/chemokine profile predictive of GF, PB samples were collected at different time points after HSCT: day $0,+3 \pm 2,+7 \pm 2,+10 \pm 2,+14 \pm 2,+30 \pm 2$ after transplantation. Validated MesoScale Discovery (MSD, Rockville, MD, USA) platform-based immunoassay was used for the quantification of IFN $\gamma$, sIL2R $\alpha$, CXCL9, CXCL10, TNF $\alpha$, IL6, IL10, and sCD163 serum levels.

\section{Bone marrow biopsy: histopathology analysis and immunofluorescence}

Bone marrow biopsies were obtained when GF was suspected. (Since BM characterization was a secondary end point of this study and BM aspiration is not routinely performed in this condition, parents/legal guardians could refuse the procedure.) Details on BM specimen preparation, histopathology analysis and immunofluorescence are reported in the Online Supplementary Appendix.

\section{Immune-phenotypic analysis}

The following monoclonal antibodies (mAbs) were used: antiCD3, CD4, CD8, CD25, CD27, CD28, CD45RA, CD45RO, CD56, CD57, CD62L, CD95, CD127, CD137, CD197, CD223 (Lag3), CD279 (PD1), and CD366 (TIM3) (BD Biosciences, NJ, Biolegend, CA and Affymetrix, CA, USA).

\section{In vivo murine model of hematopoietic stem cell transplantation rejection}

C57BL/6 Ifngr $1^{-/}$mice were used as recipient, while C57BL/6 Ifngr $1^{+/+}$were used as donor. All animal experiments were performed in accordance with the Swiss animal protection law. Details on experiments are reported in the Online Supplementary Appendix.

\section{Emapalumab administration in compassionate use to hemophagocytic lymphohistiocytosis patients experiencing graft failure}

Emapalumab (previously known as NI-0501), a fully human anti-IFN $\gamma$ monoclonal antibody, was administered on a CU basis (after local ethical committee approval) to three patients affected by HLH who experienced GF after a first TCD HSCT from a partially-matched family donor (PMFD) with the aim of preventing flares of HLH and a second GF. The drug was administered by 1-hour intravenous infusion twice a week until sustained donor engraftment or GF. The dose varied between 1 and $6 \mathrm{mg} / \mathrm{kg}$, based on pharmacokinetic data.

Additional methods are presented in the Online Supplementary Appendix.

\section{Statistical analysis}

Unless otherwise specified, quantitative variables were reported as Mean \pm Standard Error of Mean (SEM); categorical variables were expressed as absolute value and percentage. Clinical characteristics of patients were compared using the $\chi^{2}$ test or Fisher exact test for categorical variables, while the Mann-Whitney rank sum test or the Student $t$-test (two-sided) was used for continuous variables, as appropriate. For multiple comparison analyses, statistical significance was evaluated by a repeated measure ANOVA test, followed by a Log-rank (Mantel-Cox) test for multiple comparisons. 


\section{Results}

\section{Patients' characteristics}

During the study period, 15 consecutive patients who experienced GF were eligible for the study. Most of them were affected by non-malignant disorders characterized by a high risk of GF (e.g. SAA and HLH) and received a TCD allograft from a PMFD. Fifteen children, matched for transplant characteristics, who had sustained donor engraftment during the same period were used as controls. Patients' and control characteristics are detailed in Table 1. Main transplant characteristics (i.e. conditioning regimen, type of donor, graft manipulation) were comparable between the two groups (except for a trend for a lower age in the GF group). Of the 15 patients experiencing GF, ten were tested for anti-HLA antibodies, which were detected in five patients (50\%). Those who had a mean fluorescence intensity (MFI) of anti-HLA antibodies $>5000$ received rituximab and underwent plasmaexchange to lower the value below the threshold of 5000 $\mathrm{MFI} ;{ }^{18}$ this treatment successfully reduced the MFI value in all cases.

Signs and symptoms of patients who either did or did not experience GF are detailed in Table 2. The most frequent sign associated with GF was fever, occurring at a median time of six days from the infusion of the graft (range 1-16 days). Moreover, both lactate dehydrogenase (LDH) and ferritin increased in many patients $(80 \%$ and $46.7 \%$, respectively); these laboratory findings appeared late after HSCT (at a median of 11 and 10 days, respectively). All patients received steroids in an attempt to avoid GF, without benefit. Chimerism analysis performed on $\mathrm{PB}$ showed only recipient cells in all GF cases, while in all controls but one, who showed mixed chimerism, only donor-origin cells were found.

\section{Cytokine/chemokine profile}

Kinetics of IFN $\gamma$, CXCL9, IL10 and IL2R $\alpha$ serum levels are shown in Figure 1A-D, while serum levels of TNF $\alpha$, CXCL10, sCD163 and IL6 are shown in Figure 2A-D. Serum levels of these cytokines/chemokines differed between patients experiencing GF and controls, starting from the first days after the infusion of the graft. Notably, for IFN $\gamma$, CXCL9, IL10 and TNFa, this difference became
Table 1. Characteristics of patients who either did or did not experience graft failure (GF).

\begin{tabular}{|c|c|c|c|}
\hline & GF patients (\%) & Controls (\%) & $P$ \\
\hline Total & $15(100)$ & $15(100)$ & \\
\hline $\begin{array}{l}\text { Gender } \\
\text { Female } \\
\text { Male }\end{array}$ & $\begin{array}{l}9(60) \\
6(40)\end{array}$ & $\begin{array}{l}10(66.5) \\
5(33.5)\end{array}$ & 0.27 \\
\hline $\begin{array}{l}\text { Age at transplant, years } \\
\text { (median and range) }\end{array}$ & $2.6(0.3-16.3)$ & $8.1(0.9-18)$ & 0.1 \\
\hline $\begin{array}{l}\text { Disease } \\
\text { SAA } \\
\text { HLH } \\
\text { AML } \\
\text { ALL } \\
\text { Erythroid disorders } \\
\text { CAMT } \\
\text { Metabolic disorders* } \\
\text { Osteopetrosis } \\
\text { PID }\end{array}$ & $\begin{array}{l}4(27) \\
3(20) \\
1(6.5) \\
1(6.5) \\
1(6.5) \\
1(6.5) \\
2(13.5) \\
1(6.5) \\
1(6.5)\end{array}$ & $\begin{array}{l}4(27) \\
0 \\
2(13.5) \\
3(20) \\
4(27) \\
0 \\
1(6.5) \\
0 \\
1(6.5)\end{array}$ & 0.38 \\
\hline $\begin{array}{l}\text { Type of transplant } \\
\text { TCD haploidentical } \\
\text { MUD } \\
\text { UCBT }\end{array}$ & $\begin{array}{l}13(87) \\
1(6.5) \\
1(6.5)\end{array}$ & $\begin{array}{l}10(66.5) \\
5(33.5) \\
0\end{array}$ & 0.13 \\
\hline $\begin{array}{l}\text { Source of stem cells } \\
\text { PBSC } \\
\text { BM } \\
\text { Cord blood }\end{array}$ & $\begin{array}{l}13(87) \\
1(6.5) \\
1(6.5)\end{array}$ & $\begin{array}{l}11(73) \\
4(27) \\
0\end{array}$ & 0.22 \\
\hline $\begin{array}{l}\text { Conditioning regimen } \\
\text { TBI-based } \\
\text { Busulfan-based } \\
\text { Treosulfan-based } \\
\text { Other regimens }\end{array}$ & $\begin{array}{l}0 \\
8(53) \\
3(20) \\
4(27)\end{array}$ & $\begin{array}{l}2(13.5) \\
10(66.5) \\
2(13.5) \\
1(6.5)\end{array}$ & 0.23 \\
\hline $\begin{array}{l}\text { Donor/recipient pair } \\
\text { sex mismatch } \\
\text { Yes } \\
\text { No }\end{array}$ & $\begin{array}{l}4(27) \\
11(73)\end{array}$ & $\begin{array}{l}9(60) \\
6(40)\end{array}$ & 0.14 \\
\hline
\end{tabular}

SAA: severe aplastic anemia; HLH: hemophagocytic lymphohistiocytosis: AML: acute myeloid leukemia;ALL: acute lymphoblastic leukemia; CAMT: congenital amegakaryocytic thrombocytopenia; PID: primary immunodeficiency; TCD: T-cell depleted; MUD: matched unrelated donor; UCBT: unrelated cord blood transplant; PBSC: peripheral blood stem cells; BM: bone marrow; TBI: total-body irradiation; MLD: metachromatic leukodystrophy; MPS: mucopolysaccharidosis;ALD: adrenoleukodistrophy; DBA: Diamond-Blackfan anemia; * 1 case each of MLD and MPS-I in GF group; 1 case of ALD among controls; ${ }^{5} 1$ case of Thalassemia in GF group; 2 cases of Thalassemia and 2 of DBA among controls; ${ }^{\ddagger} 1$ case of combined immunodeficiency in GF group; 1 case of autosomal recessive hyper-IgE syndrome among controls. SAA: severe aplastic anemia.

Table 2. Signs and symptoms of patients who experienced graft failure (GF).

\begin{tabular}{|c|c|c|c|c|c|c|c|}
\hline Signs/symptoms & $\begin{array}{l}\text { GF patients } \\
\text { (total=15) }\end{array}$ & Percentage & $\begin{array}{l}\text { Median day } \\
\text { of onset }\end{array}$ & Range & $\begin{array}{l}\text { Controls } \\
\text { (total=15) }\end{array}$ & Percentage & $P$ \\
\hline Fever & 13 & 86.7 & 6 & $1-16$ & 7 & 46.7 & 0.02 \\
\hline Increase of ferritin serum levels & 12 & 80.0 & 11 & $4-20$ & 2 & 13.3 & 0.0007 \\
\hline Hemophagocytosis^ & $5^{\wedge}$ & $71.4^{\wedge}$ & & & $0^{\S}$ & 0 & 0.02 \\
\hline $\begin{array}{l}\text { Hypertriglyceridemia/ } \\
\text { hypofibrinogenemia }\end{array}$ & 7 & 46.7 & 12 & $4-16$ & 2 & 13.3 & N.S. \\
\hline Increase of LDH serum levels & 7 & 46.7 & 10 & $8-17$ & 1 & 6.7 & 0.03 \\
\hline Splenomegaly & 5 & 33.3 & 11 & $8-13$ & 0 & 0 & 0.04 \\
\hline Skin rash & 3 & 20.0 & 10 & $10-19$ & 2 & 13.3 & N.S. \\
\hline \multicolumn{8}{|l|}{ Other } \\
\hline Bradicardia & 1 & 6.7 & 9 & NA & 0 & 0 & N.S. \\
\hline
\end{tabular}

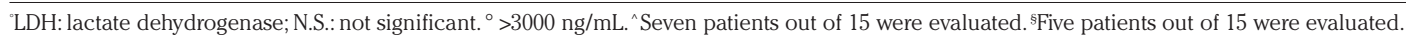




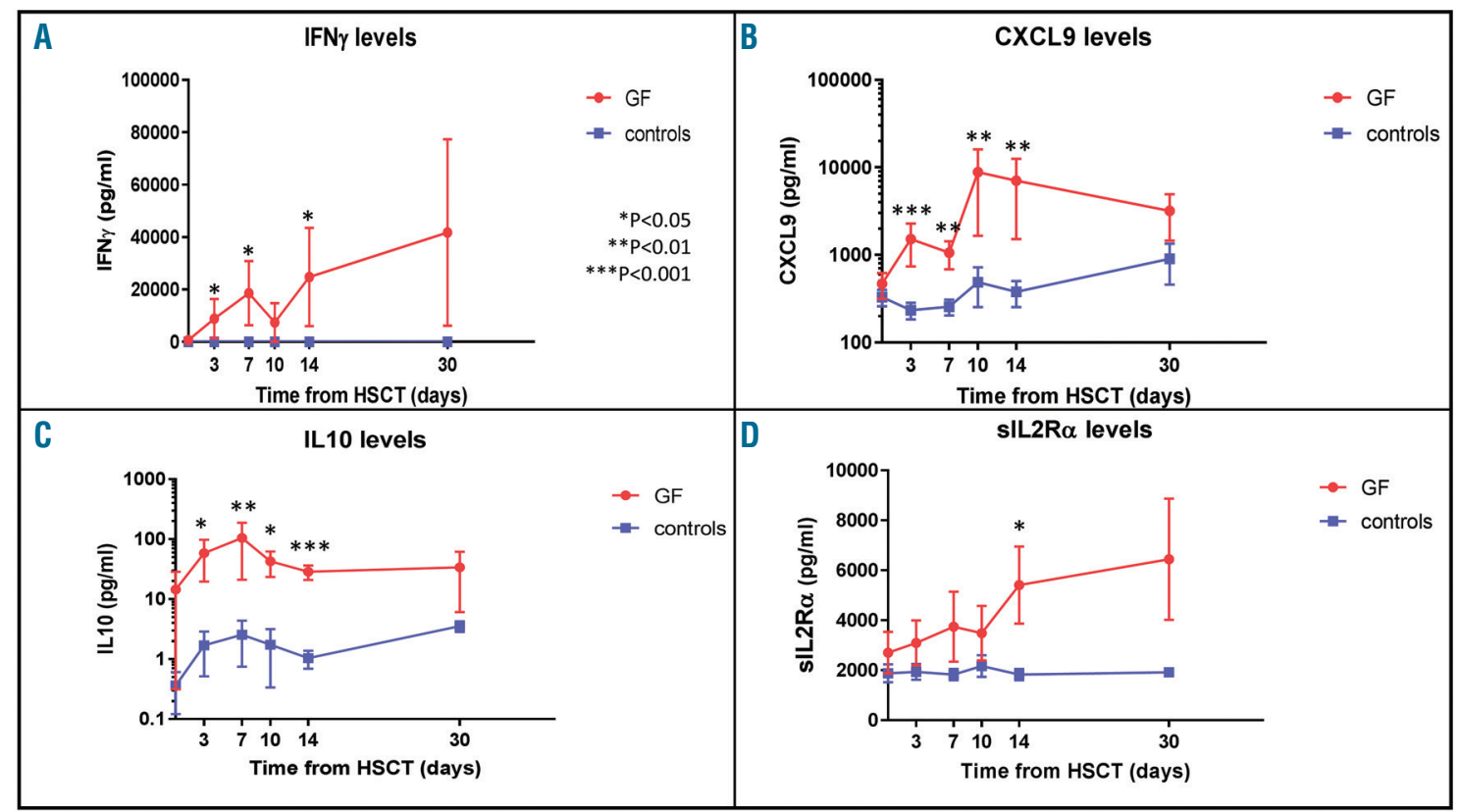

Figure 1. Cytokine/chemokine profile. Serum levels of interferon (IFN)- $\gamma$ (A), CXCL9 (B), CXCL10 (C), and sIL2R $($ D) in patients who either did (red line) or did not (blue line) experience graft failure (GF). All graphs represent Mean and Standard Error of Mean for each variable. HSCT: hematopoietic stem cell transplantation.

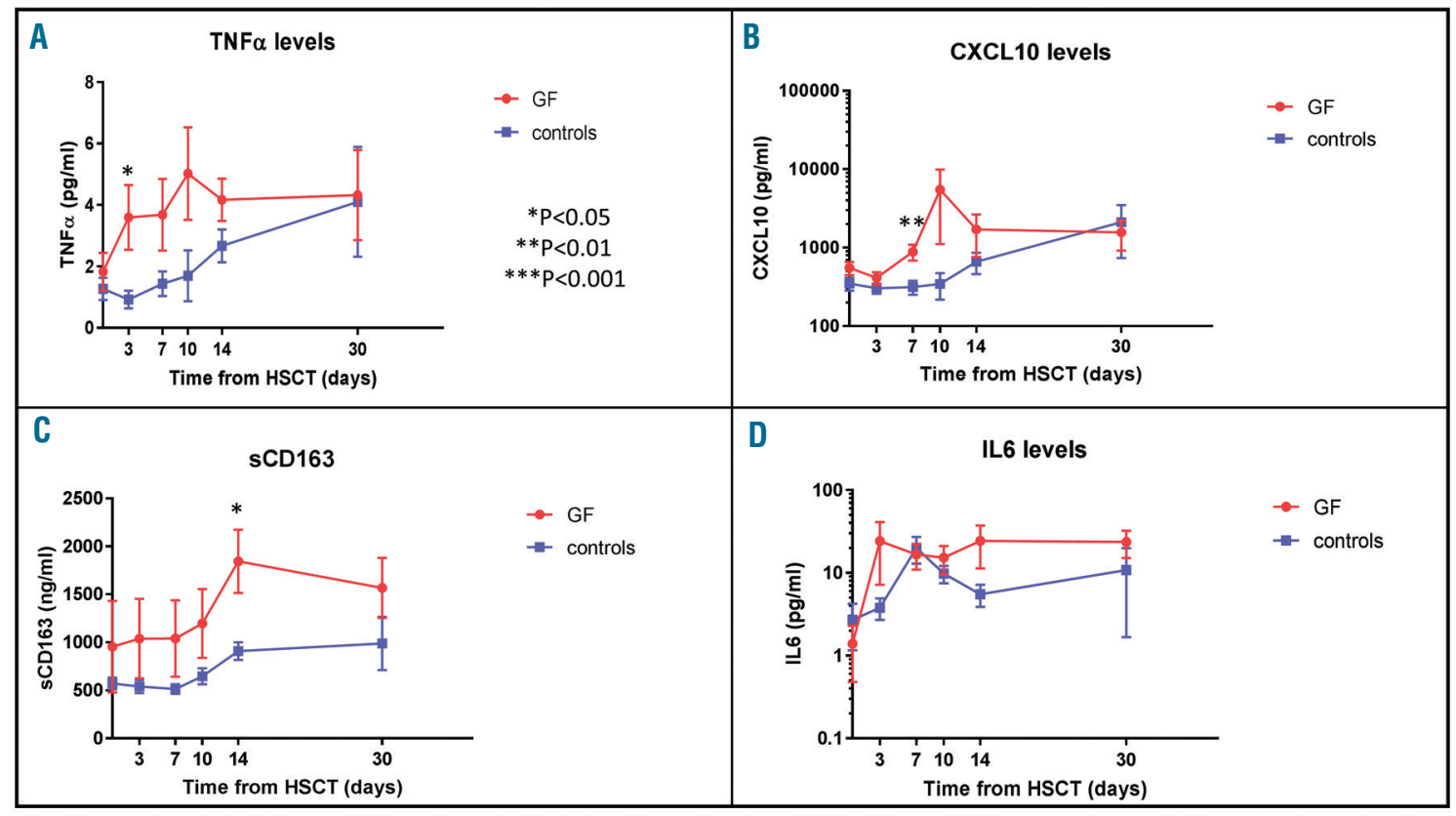

Figure 2. Cytokine/chemokine profile. Serum levels of TNF $\alpha$ (A), CXCL10 (B), sCD163 (C), IL6 (panel D). Red line: patients who experience graft failure (GF); blue line: controls. All graphs represent Mean and Standard Error of Mean for each variable. HSCT: hematopoietic stem cell transplantation. 
statistically significant already at day +3 after HSCT. In particular, mean IFN $\gamma$ levels at day +3 were $8859 \pm 7502$ $\mathrm{pg} / \mathrm{mL}$ in GF patients versus $0 \mathrm{pg} / \mathrm{mL}$ in controls $(P=0.03)$; CXCL9 levels were $1514.0 \pm 773 \mathrm{pg} / \mathrm{ml}$ versus $233.6 \pm 50.1$ $\mathrm{pg} / \mathrm{mL}(P=0.0006) ;$ IL10 levels were $58.8 \pm 39.1 \mathrm{pg} / \mathrm{mL}$ versus $1.7 \pm 1.1 \mathrm{pg} / \mathrm{mL}(\mathrm{P}=0.01)$; TNF $\alpha$ levels were $3.5 \pm 1.0$ $\mathrm{pg} / \mathrm{mL}$ versus $0.9 \pm 0.2 \mathrm{pg} / \mathrm{mL}(P=0.02)$. In this cohort, receiver operating characteristics (ROC) analysis on CXCL9 levels at day +3 showed an area under the curve (AUC) of 0.905 [95\% Confidence Interval (CI) 0.709 0.987; $P<0.0001$ ] (Online Supplementary Figure S1); a cut-off value of $274.5 \mathrm{pg} / \mathrm{mL}$ had a sensitivity of $88.89 \%$ and a specificity of $78.57 \%$. The ROC analysis of other markers, which were significantly increased at day +3 showed an AUC of 0.802 for TNF $\alpha$ (95\%CI: 0.566-0.944; $P=0.006)$, of 0.756 for IL10 (95\% CI: $0.529-0.912 ; P=0.011)$ and of 0.682 for IFN $\gamma$ (95\% CI: 0.471-0.849; $P=0.017)$.

Since primary HLH patients commonly present increased IFN $\gamma$ and its related chemokines serum levels during disease reactivation/flare (that is frequent after failure of $\mathrm{HSCT}^{19}$ ), we performed additional analyses excluding this subset of patients in order to validate the data in disorders other than HLH. Even after excluding HLH patients, CXCL9 and IL10 serum levels remained significantly higher in patients experiencing GF in comparison with controls (Online Supplementary Figure S2).

\section{Activation of macrophages and T lymphocytes characterizes graft failure in allogeneic hematopoietic stem cell transplantation}

Bone marrow biopsies were obtained at time of GF in seven patients and were compared to those of five controls (obtained in a similar time period, i.e. between 2 and 3 weeks after HSCT). In all GF patients, evaluation of BM morphology showed different stages of GF with reduced cellularity (Figure 3A and Online Supplementary Figure S3A and $B$ ) as compared to patients with sustained donor engraftment (Online Supplementary Figure S4A). In GF patients, the percentage of myelocytes and erythroid precursors was reduced compared to controls (Figure 3B). Erythroid colonies were markedly smaller, with a higher percentage of premature erythroid cells. The megakaryocytic lineage was well represented in all GF cases, but with irregular distribution (Figure 3C). In several areas of the specimens, a remarkable number of apoptotic cells partially grouped in clusters was observed (Figure 3D). All biopsies showed stromal damage resulting in edema (Figure 3E). While the total number of $\mathrm{CD}^{+} 8^{+}$ macrophages was comparable between GF patients and controls (Figure 4A), significantly higher percentages of $\mathrm{CD}^{+} 8^{+}$and $\mathrm{CD} 163^{+}$macrophages, with cellular fragments, erythrocytes and lipid vacuoles in their cytoplasm, (indicating activation and phagocytic activity) (Figure $3 \mathrm{~F}$ and G and Online Supplementary Figure S3C and D), were observed in comparison to controls [median $80 \%$ (range $30-100 \%$ ) vs. $0 \%$ (range $0-5 \%$ ); $P<0.0001$ ] (Figure $4 \mathrm{~B}$ and Online Supplementary Figure $S 4 B$ and $C$ ). In all analyzed samples from GF patients, a significant increase in $T$ lymphocytes (Figures $3 \mathrm{H}$ and $4 \mathrm{~A}$ and Online Supplementary Figure $S 3 G)$, with a predominance of $\mathrm{CD}^{+}$cytotoxic $\mathrm{I}$ cells, expressing perforin, Granzyme B and TIA-1 (Figures 3I and J and 4A and Online Supplementary Figure S5) was observed. The Online Supplementary Appendix provides further details.

\section{Polyclonal T-cell pattern with predominant CD8 effector memory phenotype effector memory phenotype}

In order to better characterize the role of $\mathrm{T}$ lymphocytes in GF, the TCR repertoire was initially analyzed in the $\mathrm{CD}^{+}$population, showing a polyclonal distribution of the $\mathrm{V} \beta$ chains (Online Supplementary Figure S6). Then, we
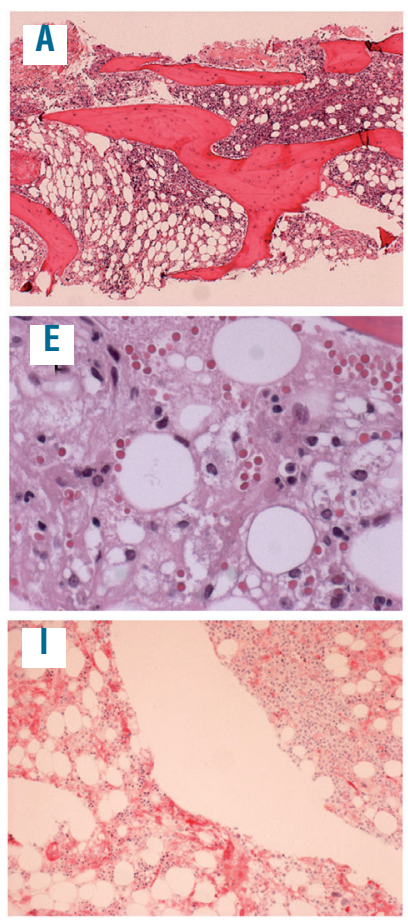
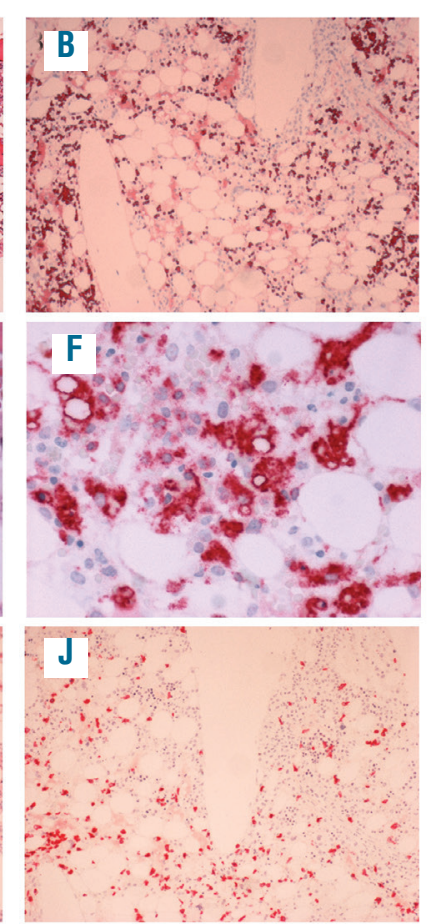
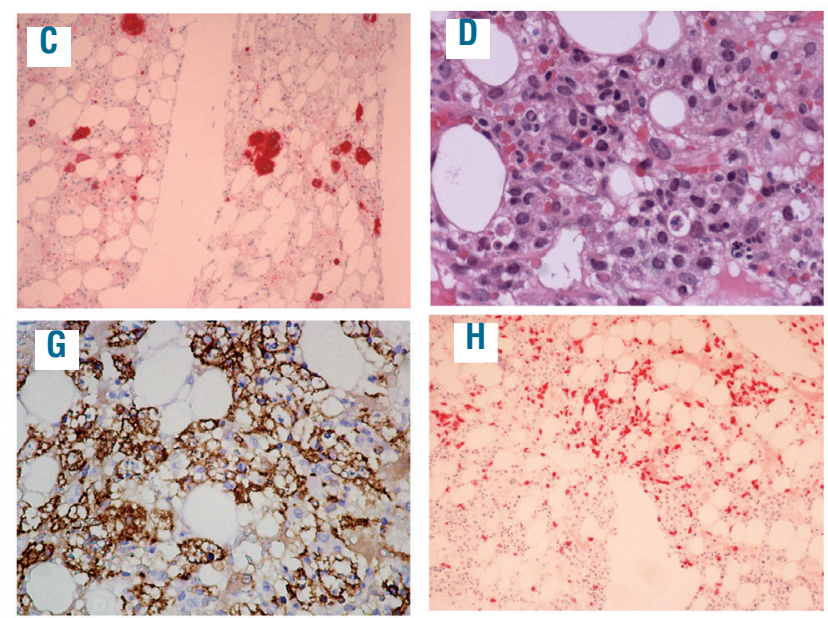

Figure 3. Immunohistochemistry evaluation of bone marrow (BM) specimens in a patient experiencing graft failure (Pt \#4). (A) Hematoxylin \& eosin (H\&E) staining of a BM specimen at $4 \mathrm{X}$ magnification. (B) Evaluation of erythroid colony spreading by glycophorin staining (10X). (C) Megakaryocyte distribution evaluated by CD61 expression (10X). (D) H\&E staining at 40X showing apoptotic events. (E) H\&E staining revealing stromal damage and edema development (40X). Characterization of the macrophage population by CD68 (F) and CD163 (G) staining (4OX). Characterization and distribution of T lymphocytes by analysis of CD3 (H), CD4 (I), and CD8 (J) expression (10X). 
A

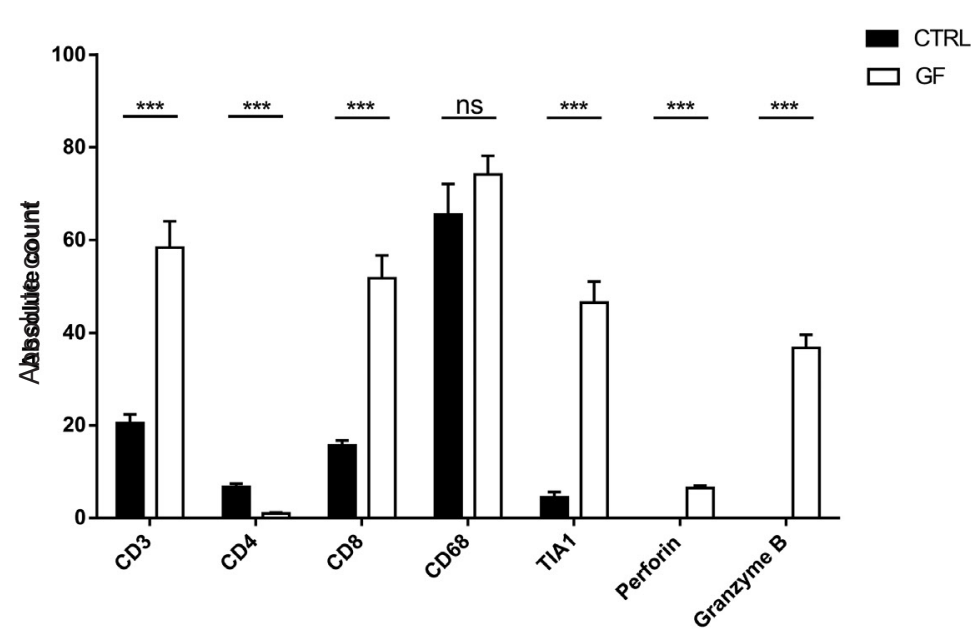

B

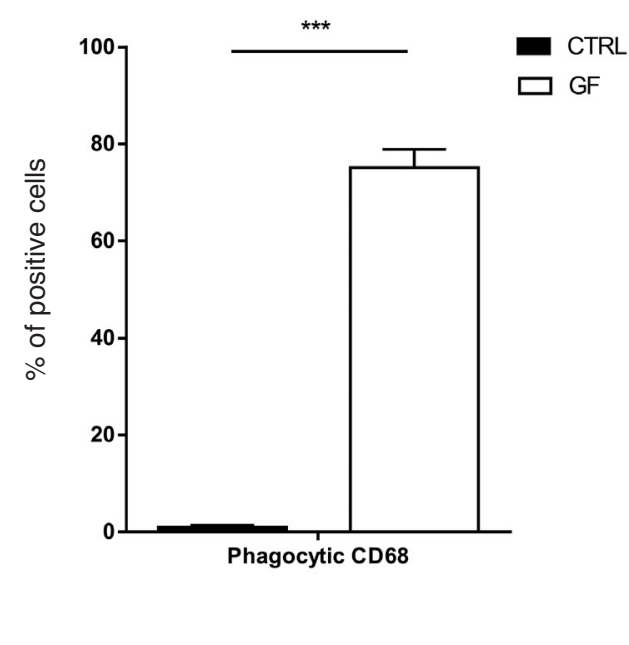

Figure 4. Immunohistochemistry characterization of bone marrow (BM) in patients who either did or did not experience graft failure (GF). (A) Comparison of absolute number of $\mathrm{CD}^{+}, \mathrm{CD}^{+}, \mathrm{CD}^{+}, \mathrm{CD}^{+} 8^{+}, \mathrm{TIA}^{-1}{ }^{+}$, perforin ${ }^{+}$and granzyme ${ }^{+}$cells in $\mathrm{BM}$ of GF patients and controls (CTRL). The total number of positive cell for each marker was counted in five fields per sample under 20 -fold magnification and reported as Mean+Standard Deviation. (B) Percentages of CD68 ${ }^{+}$cells with hemophagocytic activity (i.e. showing cellular fragments, erythrocytes and lipid vacuoles in their cytoplasm) in BM of GF patients and CTRL. $* P<0.05$; $* * P<0.01$; $* * * P<0.001$.

extended our analysis on BM-infiltrating lymphocytes through flow-cytometry in both controls and GF patients. Regarding $\mathrm{NK}\left(\mathrm{CD}^{+} 6^{+} \mathrm{CD} 3^{-}\right)$and $\gamma \delta \quad \mathrm{T}$ cells $\left(\mathrm{CD}^{+} / \mathrm{CD}^{-} / \mathrm{CD}^{-}\right)$no difference was observed between the two patient groups (data not shown). By contrast, in the $\alpha \beta$ T-cell subset, the analysis revealed a significant difference in both CD4 $(58.9 \% \pm 13.4 \%$ vs. $7.6 \% \pm 7.3 \%$, controls vs. GF patients) and CD8 $(25.9 \% \pm 6.1 \%$ vs. $66.5 \% \pm 18.2 \%$, controls vs.GF patients) subsets $(P<0.0001$ and $P=0.0018$, respectively) (Figure 5A). We further characterized both $\mathrm{CD} 4^{+}$and $\mathrm{CD} 8^{+}$populations for the expression of memory markers. While no significant difference was detected in the $\mathrm{CD} 4^{+}$subpopulation, the $\mathrm{CD} 8^{+}$subset displayed a significant enrichment of effector memory $\mathrm{T}$ cells (EfM) (CD45RO+CCR7-) (40.3 $\pm 24.6 \%$ vs. $20.7 \% \pm 7.3 \%$, GF patients vs. CTRL patients; $P=0.034$ ) (Figure $5 \mathrm{~B}$ and $\mathrm{C}$ ) and a significant reduction of the naïve subset $\left(\mathrm{CD}^{2} 5 \mathrm{RA}^{+} / \mathrm{CCR}^{+}\right)(18.6 \% \pm 16.6 \%$ vs. $28.6 \% \pm 12.1 \%$, GF patients vs. controls; $P=0.014)$. See Online Supplementary Appendix for further details.

\section{Increasing expression of activation and exhaustion markers on T cells during graft failure}

We evaluated the expression of several activation and exhaustion markers on infiltrating cells. As expected, in patients experiencing GF, both $\mathrm{CD} 4^{+}$and $\mathrm{CD}^{+}$cells displayed a significant activation profile, as demonstrated by the overexpression of CD95 $(69.2 \% \pm 23.0 \%$ vs. $93.9 \% \pm 6.9 \%$ and $57.9 \% \pm 27.2 \%$ vs. $98.35 \% \pm 2.0 \%$, controls vs. GF patients, respectively; $P=0.021$ and $P=0.002$ ) (Figure 5D) and downregulation of CD127 (recently shown to be associated with prolonged T-cell receptor stimula$\left.\operatorname{tion}^{20}\right)$ on the proliferating $\mathrm{CD}^{+}$cells $(69.3 \% \pm 16.9 \%$ vs. $37.9 \% \pm 18.8 \%$, controls vs. GF patients, respectively; $P=0.014$ ) (Figure 5E). The expression of several exhaustion and senescence markers confirmed the status of prolonged activation of $\mathrm{T}$ lymphocytes located in the BM of GF patients, such as the upregulation of $\mathrm{CD} 57$ (CD57':
$10.2 \% \pm 10.5 \%$ vs. $37.4 \% \pm 12.4 \%$ and $34.7 \% \pm 17.3 \%$ vs. $68.0 \% \pm 18.8 \%$ controls vs. GF patients in CD4 and CD8 respectively; $P=0.003$ and $P=0.011$ ) (Figure 5F). See Online Supplementary Appendix for further details.

\section{Interferon- $\gamma$ drives rejection of donor cells in Ifngr $1^{-/-}$ mice}

In order to understand if the sole IFNy-inhibition would be sufficient to prevent GF, we used an established mouse model of GF. ${ }^{13}$ As previously reported by Rottman et al. ${ }^{13}$ the infection of Ifngr $1^{-1}$ mice with Bacillus CalmetteGuérin (BCG) resulted in a rapid increase of circulating IFNy levels reaching a concentration of $11,000 \mathrm{pg} / \mathrm{mL}$ on day 20 post-infection (Figure 6A). HSCT performed at day 21 , i.e. at the peak of IFN $\gamma$ levels, resulted in poor chimerism as only $5 \%$ of the Ifngr $1^{+/+}$donor cells engrafted in the BCG-infected Ifngr 1 - recipient mice. After day 21 post-BCG infection, serum IFN $\gamma$ levels gradually decreased to a steady state level of approximately $100 \mathrm{pg} / \mathrm{mL}$. This decrease in IFN $\gamma$ serum levels correlated with an increase in chimerism as the Ifngr $1^{-/}$recipient mice exhibited 19\% HSC engraftment of donor cells at day 84 (Figure 6A). For further assessing the role played by IFN $\gamma$ in GF, BCGinfected Ifngr $1^{-}$recipient mice were given a neutralizing IFN $\gamma$ mAb, XMG1.2, pre- and post-HSCT. Neutralization of IFN $\gamma$ improved engraftment in BCG-infected Ifngr1 recipient mice because, at three months after the allograft, $45 \%$ of the lymphocytes were of donor origin (i.e. Ly5.1 positive), as compared to $19 \%$ in isotype control-treated mice (Figure $6 \mathrm{~B})$. In order to assess IFN $\gamma$ activity and ensure neutralization by XMG1.2, the IFNy-dependent chemokine CXCL9 was measured. A decrease in CXCL9 serum levels during the XMG1.2 treatment was observed, confirming IFN $\gamma$ neutralization in contrast to isotype control-treated mice (Figure 6C). Once XMG1.2 treatment was interrupted, at day 42 post-BCG infection, a gradual increase in CXCL9 serum levels was observed, indicating restoration of IFN $\gamma$ activity. 
A

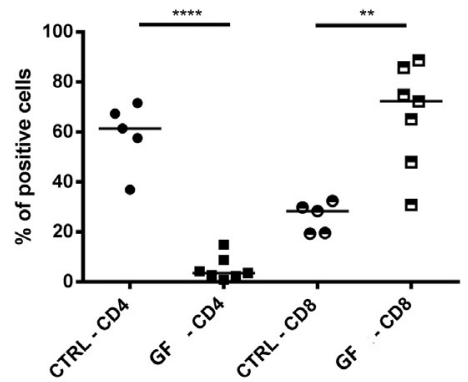

D

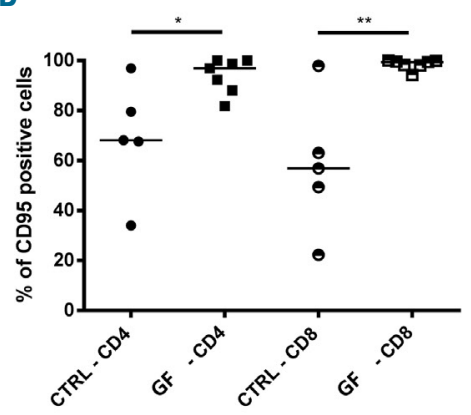

B

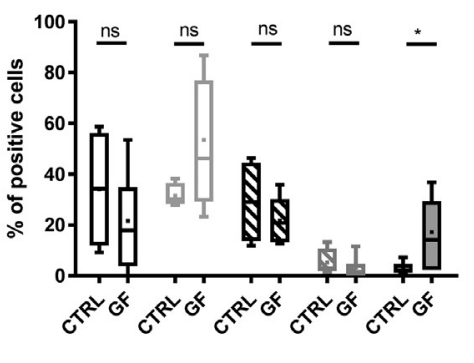

E

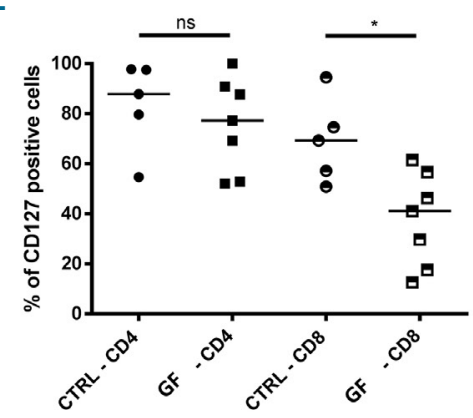

c
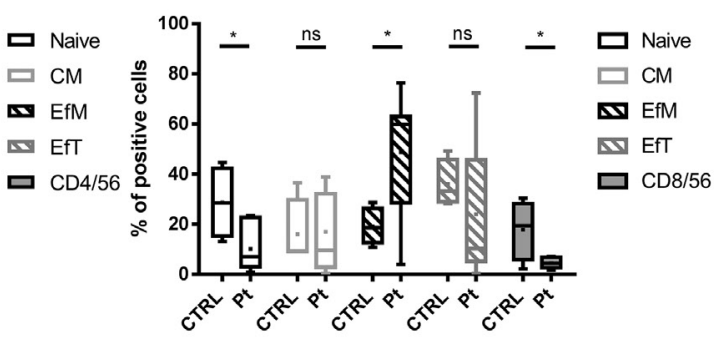

F

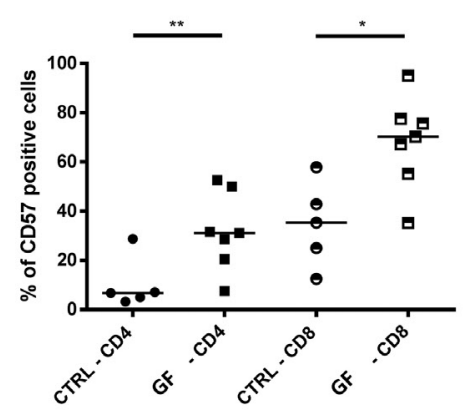

Figure 5. Immuno-characterization of the T lymphocytes present in bone marrow aspirates of patients who either did or did not experience graft failure (GF). (A) Flow cytometry analysis of $\mathrm{CD}^{+}$and $\mathrm{CD}^{+}$population in patients with GF and controls (CTRL). Distribution of naïve (CD45RA $/ \mathrm{CCR} 7^{+}$), central memory

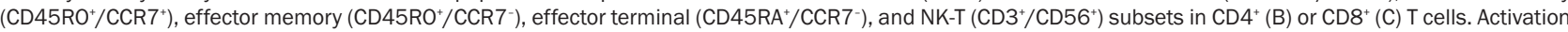
and exhaustion profile in both the CD4 $4^{+}$and $C D 8^{+}$population by the analysis of CD95 (D), CD127 (E), and CD57 (F). (A, D, E, and F) Each patient or CTRL is represented by a symbol and a horizontal line marks the median. (B and $\mathrm{C}$ ) The average $(+)$ and Median \pm Standard Deviation are shown. $* P<0.05$; $* \star P<0.01$; $* * * P<0.001 ; * * * * P<0.0001$.

\section{Emapalumab administration to patients after} hematopoietic stem cell transplantation failure

Three patients with primary HLH who experienced GF together with disease reactivation after a first TCD HSCT from a PMFD were treated with emapalumab both before and after the second HSCT (details are reported in Online Supplementary Table S1). For all these patients, the use of the other parent as a donor was not possible because of non-eligibility due to viral hepatitis. The CU of emapalumab was requested and obtained with the objective of controlling, without the use of myelosuppressive drugs other than those used in the conditioning regimen, $\mathrm{HLH}$ reactivation before and after a second HSCT. Emapalumab was administered at doses of $1-6 \mathrm{mg} / \mathrm{kg}$ every three days. Drug infusions were well tolerated and no significant safety event occurred. Two patients engrafted, while one rejected also the second HSCT without, however, experiencing a new HLH flare. This patient was successfully rescued with a third HSCT employing an unrelated cord blood (UCB) unit (notably, she received emapalumab until 3 days before UCB infusion). Remarkably, the two patients who engrafted upon treatment with emapalumab had very low levels of CXCL9 (i.e. below $102 \mathrm{pg} / \mathrm{mL}$ ), indicating IFN $\gamma$ neutralization, while this was not the case for the third patient at the time of the second transplant rejection. All these three patients are currently alive and disease-free, with a follow up of 24 , 23 and 21 months, respectively.

\section{Discussion}

Diagnosis and treatment of GF in HSCT recipients remain challenging. Indeed, sign and symptoms (e.g. fever, increase in LDH or ferritin serum levels) associated with this transplant complication are non-specific; moreover, re-transplantation, although associated with relevant risk of tissue-toxicity and infections, represents the treatment of choice, since steroids and other immunosuppressive drugs are usually ineffective for rescuing these patients. ${ }^{2}$ In this study, we investigated humoral and cellular features of GF occurring after allogeneic HSCT in children, documenting a pivotal role played by IFN $\gamma$ in the pathophysiology of this complication. Apart from the indirect evidence provided by the observation of very high rates of primary and secondary rejection after HLA-identical HSCT in patients with IFN $\gamma$-receptor 1 deficiency, ${ }^{21}$ currently available clinical data about the role of IFN $\gamma$ in GF in humans remain limited. Interestingly, we found that GF is characterized by the same clinical (including high-grade fever, hepato/splenomegaly, hemophagocytosis in BM) (2,23 $^{2,2}$ and laboratory (i.e. increased ferritin, IFN $\gamma$, CXCL9, CXCL10, sCD163 and sIL-2R $\alpha$ levels) ${ }^{24-28}$ features found in patients with $\mathrm{HLH}$, where a central role of IFNy has been shown. ${ }^{29}$

Our data indicate that IFN $\gamma$ levels, and even more CXCL9 levels measured in PB, can predict GF with high sensitivity and specificity already at day +3 after graft 

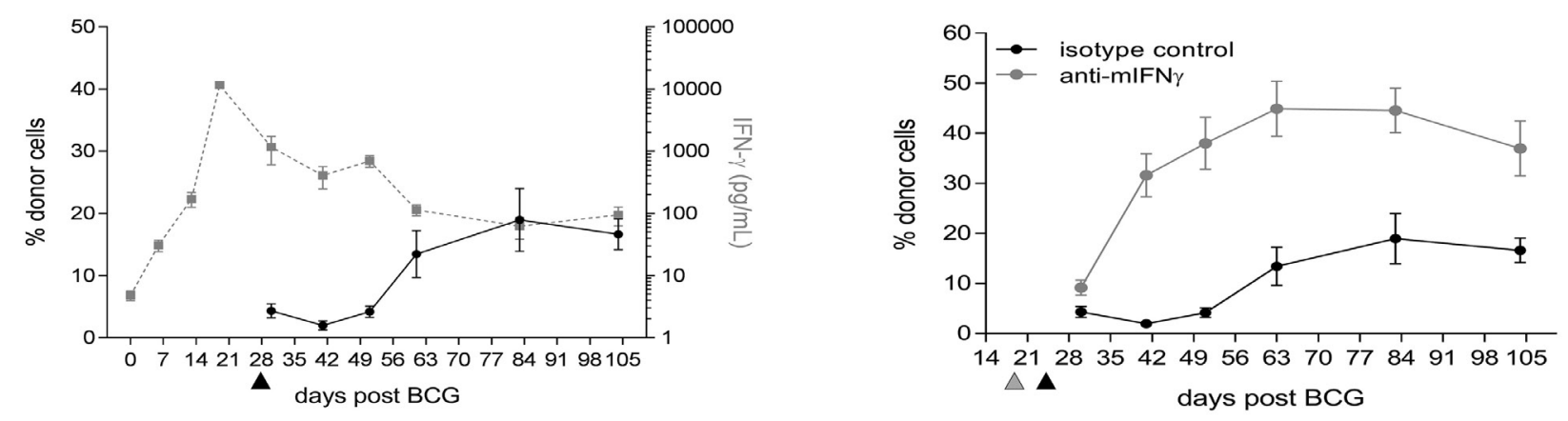

C

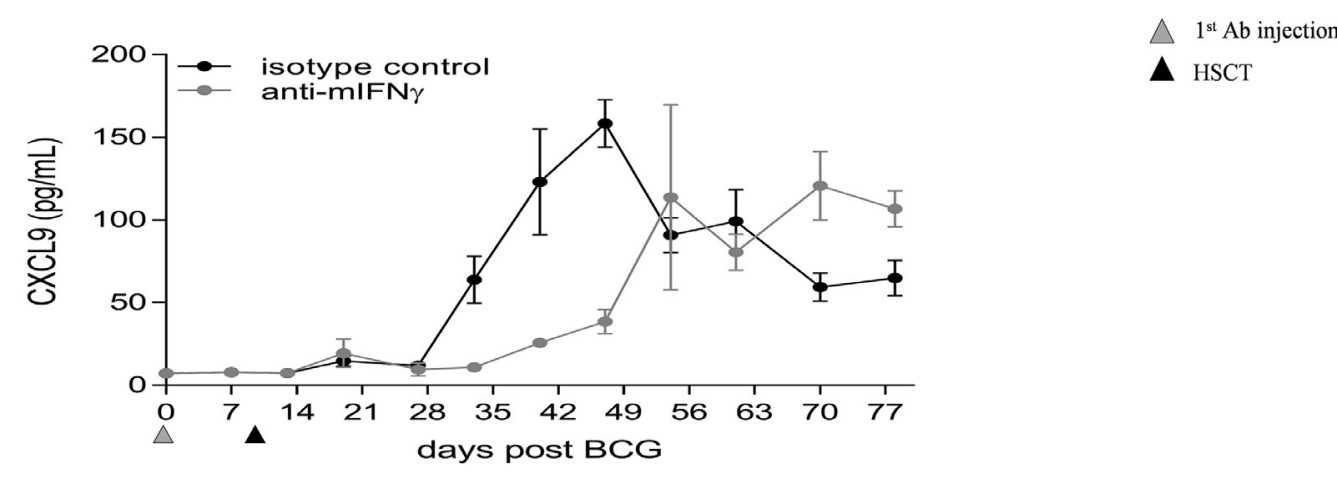

Figure 6. Successful hematopoietic stem cell transplantation (HSCT) chimerism in interferon (IFN)-yR1 mice correlates with low IFNy activity; circulating CXCL9 levels is a biomarker of in vivo IFN $\gamma$ activity. Ifngr $1 \%$ mice (expressing the Ly5.2 congenic marker) were intravenously (i.v.) infected with $1,106 \mathrm{CFU}$ of Bacillus Calmette-Guérin (BCG) (strain Pasteur 1173P2). After 14, 20, 28, 35 and 42 days mice were treated i.v. with $100 \mathrm{mg} / \mathrm{kg}$ of an isotype control ( $\mathrm{n}=5$ ) or the antimIFN $\gamma$, XMG1.2 ( $n=5)$. At day 21, mice were infused with bone marrow from Ifngr ${ }^{+/+}$mice, expressing the Ly5.1 marker, after mild irradiation (550 rads). Chimerism, assessed by determining the surface expression of Ly5.1 and Ly5.2 on lymphocytes, was analyzed by flow cytometry at different time points after HSCT treatment. IFN $\gamma$ levels were quantified at different time points by ELISA using the Luminex technology. (A) Graph represents the super-imposition of the chimerism (black straight line) and the IFN $\gamma$ levels (gray dotted line) in the isotype control treated mice. (B) Graph represents the chimerism determined in mice treated with the isotype control (black straight line) or with the XMG1.2 (gray straight line) mAbs. (C) Ifngr1\% mice were i.v. infected with 1.106 CFU of BCG (strain Pasteur 1173P2). After 14, 20, 28, 35 and 42 days mice were treated i.v. with $100 \mathrm{mg} / \mathrm{kg}$ of an isotype control (black straight line; $\mathrm{n=5}$ ) or the anti-mIFN $\gamma, \mathrm{XMG1.2}$ (gray straight line; $\mathrm{n}=5$ ). At day 21, mice were transplanted with bone marrow from Ifngr ${ }^{+/+}$mice, expressing the Ly5.1 marker, after mild irradiation (550 rads). At different time points post-BCG infection, circulating CXCL9 levels were quantified by ELISA using the Luminex technology. Ab: antibody.

infusion, while signs and symptoms of GF appear only later (see Table 2). Indeed, the current proposed risk score for GF determined on day +21 after HSCT, based on eight patient and transplant variables, showed good specificity, but low sensitivity. ${ }^{1}$ The high accuracy of CXCL9 in predicting GF, as indicated by the AUC of 0.905 , renders this chemokine an ideal "candidate biomarker", as stated by the 2014 National Institutes of Health consensus on biomarkers. ${ }^{30,31}$ CXCL9, also known as monokine induced by $\gamma$-interferon (MIG), is a chemokine specifically induced by IFN $\gamma,{ }^{32}$ and represent the most sensitive and specific of the soluble factors we analyzed. It binds to the chemokine receptor CXCL3 expressed on naive $\mathrm{T}$ cells, Th1 $\mathrm{CD}^{+} \mathrm{T}$ cells, effector $\mathrm{CD}^{+} \mathrm{T}$ cells, as well as on NK and NKT cells, driving Th1 inflammation. Circulating CXCL9 levels have been shown to reflect the amount of IFN $\gamma$ produced in organs, such as liver and spleen, ${ }^{25}$ which are the typical target of inflammation. This strong correlation with IFN $\gamma$ produced in organs rather than in blood provides an explanation why, despite high CXCL9 serum levels, serum levels of IFN $\gamma$ were found to be low or even undetectable in a few of our GF patients. Furthermore, elevated levels of
CXCL9 have been related to graft rejection in solid organ transplantation (such as heart, kidney and lung transplantation), ${ }^{33-35}$ but, to the best of our knowledge, this is the first report demonstrating that the hyperproduction of IFN $\gamma$ in GF occurring after HSCT results in increased CXCL9 serum levels. Among other cytokines/ chemokines, we also observed increased levels of IL10, an important Th2 cytokine with anti-inflammatory properties, this finding being in agreement with the hyperproduction of this molecule recorded in patients with HLH. ${ }^{36}$

Our results are not only relevant for diagnostic purposes, but also suggest that IFN $\gamma$ is a potential therapeutic target in GF. Indeed, independently of the mechanism of IFN $\gamma$ mediated GF (i.e. direct effect on HSC or HLH-like effect), our results support the investigation of IFN $\gamma$ neutralization for prevention and/or treatment of GF in patients undergoing HSCT. The encouraging efficacy and safety data reported from the ongoing study in primary HLH with emapalumab (NI-0501), an anti-IFN $\gamma$ monoclonal antibody, ${ }^{17,37}$ provides additional support for the rationale for using this drug. ${ }^{38}$ The data we generated in the murine model of GF confirm and extend the role played by IFN $\gamma$ 
previously demonstrated by Rottman et al. ${ }^{13}$ Moreover, we also show that the sole neutralization of IFN $\gamma$, without the administration of anti-IL12 (employed in the experiments reported by Rottman et al.), ${ }_{13}^{13}$ is able to improve engraftment. The observation that decreased CXCL9 production correlates with improved HSCT chimerism provides further support to a therapeutic intervention aimed at neutralizing IFN $\gamma$-pathway signaling. Finally, the data obtained in the three patients treated on a CU basis indicate that the use of an anti-IFN $\gamma$ monoclonal antibody is safe also in a very fragile population, namely infants with a previous GF undergoing a second HSCT.

Four out of the seven patients we studied who underwent BM aspirate and biopsy showed evidence of hemophagocytosis. Indeed, it has been shown that an increased number of hemophagocytic macrophages in the BM obtained $14 \pm 7$ days after HSCT is associated with higher risk of death due to $\mathrm{GF}^{39}$ Moreover, in a cohort of adult patients receiving cord blood transplantation, GF was strictly related to the occurrence of HLH manifestations. ${ }^{23}$ Recently, in a retrospective study on peri-engraftment BM samples from 32 adult patients, Kawashima et al. proposed two histological measures, namely macrophage ratio and $\mathrm{CD}^{+}$ratio (defined as the ratio between the macrophage or $\mathrm{CD}^{+}$lymphocyte number on the total nucleated cell number), as predictors of GF at day $+14 .{ }^{15}$

Despite some preliminary studies characterizing host $T$ cell expansion in patients with $\mathrm{GF}^{15,16}$ no information is available regarding the phenotype of these cells. Our data indicate an active role of $\mathrm{T}$ lymphocytes in mediating GF. As previously reported, ${ }^{15}$ in these patients, the mononuclear infiltrate is mainly constituted by cytotoxic $\mathrm{CD}^{+}$ lymphocytes with a predominant effector memory phenotype. This population was demonstrated to be activated, proliferating and cytotoxic, expressing specific molecules, such as Granzyme B, Perforin and TIA-1, involved in target-killing, as well as various activation and proliferation markers. Interestingly, we observed that CD8 ${ }^{+}$lymphocyte expansion is predominantly polyclonal, suggesting that the immune response is directed towards several antigens and not against few immunodominant epitopes. However, a significant enrichment of certain $\beta$ clones was found. The cytopathic effect was clearly demonstrated by apoptotic cells surrounding proliferating $T$ cells, which are long-term activated, as demonstrated by the expression of several exhaustion markers. ${ }^{40,41}$ Furthermore, the remaining $\gamma / \delta$ and $\mathrm{CD}^{+} \mathrm{T}$-cell populations are similarly expressing exhaustion markers, underlying an over-stimulated environment. Notably, a particular behavior was observed in the NKT-cell population with a significant reduction of $\mathrm{CD}^{+} \mathrm{NKT}$, probably due to their activation and a significant increase of $\mathrm{CD}^{+} \mathrm{NKT}$. The role of these cells is yet to be fully elucidated, although they were shown to be able to prevent pancreatic islet transplant rejection, but also to sustain CD8 ${ }^{+}$T-cell expansion. ${ }^{42,43}$ Given these data, a treatment able to interrupt the overproduction of molecules responsible for inflammation, ${ }^{33}$ such as an anti-IFN $\gamma$, could be beneficial in this setting.

Fifty percent of tested patients had anti-HLA antibodies: all those with positivity $>5,000 \mathrm{MFI}$ received a desensitization therapy in order to lower the antibody title with the aim of reducing the risk of GF. Although we cannot exclude a role of anti-HLA antibodies in causing GF in our patients, all five positive patients showed increased values of IFN $\gamma$ and/or related cytokines after HSCT. Thus, we can hypothesize that there may be a common final pathway and/or combined action (like that reported in solid organ transplantation $)^{44}$ between humoral and cellular mechanisms sustaining GF.

Limitations of this study are the lack of a validation cohort and the relatively small number of patients included in the study. Another important limitation is that most patients experiencing GF that we report were transplanted from a PMFD after a TCD procedure (both being wellknown risk factors for GF); ; thus, our results should be further validated in other transplant settings, especially when post-transplant pharmacological graft-versus-host disease prophylaxis is used. Indeed, the use of calcineurin inhibitors or other immunosuppressive agents can modify IFN $\gamma$ (and related cytokines) secretion kinetics. ${ }^{45}$

Overall, our data suggest that immune-mediated GF may share clinical and laboratory characteristics with HLH. Besides providing evidence for further investigating the use of markers to allow a non-invasive, prompt identification of patients at high risk of developing this severe complication of HSCT, the increased serum levels of IFN $\gamma$ and CXCL9 found in GF patients provide a rationale for investigating a targeted therapy (i.e. anti-IFN $\gamma$ therapy) in this complication. We are currently designing a clinical trial on the use of emapalumab for prevention and/or treatment of GF in patients at high risk of developing this complication.

\section{Funding}

This work was supported by "Ricerca corrente" (Ministero della Salute) (PM), Investigator Grant 2015 Id. 17200 by Associazione Italiana per la Ricerca sul Cancro (AIRC) (FL) and by Novimmune SA, Switzerland.

\section{References}

1. Olsson RF, Logan BR, Chaudhury S, et al. Primary graft failure after myeloablative allogeneic hematopoietic cell transplantation for hematologic malignancies. Leukemia. 2015;29(8):1754-1762.

2. Locatelli F, Lucarelli B, Merli P. Current and future approaches to treat graft failure after allogeneic hematopoietic stem cell transplantation. Expert Opin Pharmacother. 2014;15(1):23-36.

3. Olsson R, Remberger M, Schaffer M, et al. Graft failure in the modern era of allogeneic hematopoietic SCT. Bone Marrow
Transplant. 2013;48(4):537-543.

4. Cluzeau T, Lambert J, Raus N, et al. Risk factors and outcome of graft failure after HLA matched and mismatched unrelated donor hematopoietic stem cell transplantation: a study on behalf of SFGM-TC and SFHI. Bone Marrow Transplant. 2016;51(5):687691.

5. Masouridi-Levrat S, Simonetta F, Chalandon Y. Immunological Basis of Bone Marrow Failure after Allogeneic Hematopoietic Stem Cell Transplantation. Front Immunol. 2016;7:362

6. Murphy WJ, Kumar V, Bennett M. Acute rejection of murine bone marrow allografts by natural killer cells and T cells. Differences in kinetics and target antigens recognized. J Exp Med. 1987;166(5):1499-1509.

7. Komatsu M, Mammolenti M, Jones $M$ Jurecic R, Sayers TJ, Levy RB. Antigenprimed CD8+ T cells can mediate resistance, preventing allogeneic marrow engraftment in the simultaneous absence of perforinCD95L-, TNFR1-, and TRAIL-dependent killing. Blood. 2003;101(10):3991-3999.

8. Chen J, Feng X, Desierto MJ, Keyvanfar K, Young NS. IFN- $\gamma$-mediated hematopoietic cell destruction in murine models of immune-mediated bone marrow failure. Blood. 2015;126(24):2621-2631. 
9. Chen J, Lipovsky K, Ellison FM, Calado RT, Young NS. Bystander destruction of hematopoietic progenitor and stem cells in a mouse model of infusion-induced bone marrow failure. Blood. 2004;104(6):1671-1678.

10. de Bruin AM, Demirel O, Hooibrink B, Brandts $\mathrm{CH}$, Nolte MA. Interferon- $\gamma$ impairs proliferation of hematopoietic stem cells in mice. Blood. 2013;121(18):3578-3585.

11. Lin FC, Karwan M, Saleh B, et al. IFN- $\gamma$ causes aplastic anemia by altering hematopoietic stem/progenitor cell composition and disrupting lineage differentiation. Blood. 2014;124(25):3699-3708.

12. Maciejewski J, Selleri C, Anderson S, Young NS. Fas antigen expression on CD34+ human marrow cells is induced by interferon $\gamma$ and tumor necrosis factor $\alpha$ and potentiates cytokine-mediated hematopoietic suppression in vitro. Blood. 1995;85(11): 3183-3190.

13. Rottman M, Soudais C, Vogt G, et al. IFN- $\gamma$ mediates the rejection of haematopoietic stem cells in IFN- $\gamma$ R1-deficient hosts. PLoS Med. 2008;5(1):e26.

14. Selleri C, Maciejewski JP, Sato T, Young NS. Interferon- $\gamma$ constitutively expressed in the stromal microenvironment of human marrow cultures mediates potent hematopoietic inhibition. Blood. 1996; 87(10):4149-4157.

15. Kawashima N, Terakura S, Nishiwaki S, et al. Increase of bone marrow macrophages and $\mathrm{CD} 8+\mathrm{T}$ lymphocytes predict graft failure after allogeneic bone marrow or cord blood transplantation. Bone Marrow Transplant. 2017:52(8):1164-1170.

16. Koyama M, Hashimoto D, Nagafuji K, et al. Expansion of donor-reactive host $\mathrm{T}$ cells in primary graft failure after allogeneic hematopoietic SCT following reducedintensity conditioning. Bone Marrow Transplant. 2014:49(1):110-115.

17. Jordan M, Locatelli F, Allen C, et al. A Novel Targeted Approach to the Treatment of Hemophagocytic Lymphohistiocytosis (HLH) with an Anti-Interferon $\gamma$ (IFN $\gamma$ ) Monoclonal Antibody (mAb), NI-0501: First Results from a Pilot Phase 2 Study in Children with Primary HLH. Blood. 2015; 126(23):3.

18. Ciurea SO, Thall PF, Milton DR, et al. Complement-Binding Donor-Specific AntiHLA Antibodies and Risk of Primary Graft Failure in Hematopoietic Stem Cell Transplantation. Biol Blood Marrow Transplant. 2015;21(8):1392-1398.

19. Messina C, Zecca M, Fagioli F, et al. Outcomes of Children with Hemophagocytic Lymphohistiocytosis Given Allogeneic Hematopoietic Stem Cell Transplantation in Italy. Biol Blood Marrow Transplant. 2018;24(6):1223-1231.

20. Utzschneider DT, Alfei F, Roelli P, et al. High antigen levels induce an exhausted pheno- type in a chronic infection without impairing T cell expansion and survival. J Exp Med. 2016;213(9):1819-1834.

21. Roesler J, Horwitz ME, Picard C, et al Hematopoietic stem cell transplantation for complete IFN- $\gamma$ receptor 1 deficiency: a multi-institutional survey. J Pediatr. 2004; 145(6):806-812

22. Abe Y, Choi I, Hara K, et al Hemophagocytic syndrome: a rare complication of allogeneic nonmyeloablative hematopoietic stem cell transplantation. Bone Marrow Transplant. 2002;29(9):799801.

23. Takagi S, Masuoka K, Uchida N, et al. High incidence of haemophagocytic syndrome following umbilical cord blood transplantation for adults. Br J Haematol. 2009; 147(4):543-553.

24. Bracaglia C, de Graaf K, Pires Marafon D, et al. Elevated circulating levels of interferon- $\gamma$ and interferon- $\gamma$-induced chemokines characterise patients with macrophage activation syndrome complicating systemic juvenile idiopathic arthritis. Ann Rheum Dis. 2017;76(1):166-172

25. Buatois V, Chatel L, Cons L, et al. Use of a mouse model to identify a blood biomarker for IFNy activity in pediatric secondary hemophagocytic lymphohistiocytosis. Transl Res. 2017;180:37-52.e2.

26. Henter JI, Elinder G, Soder O, Hansson M, Andersson B, Andersson U. Hypercytokinemia in familial hemophagocytic lymphohistiocytosis. Blood. 1991, 78(11):2918-2922.

27. Xu XJ, Tang YM, Song H, et al. Diagnostic accuracy of a specific cytokine pattern in hemophagocytic lymphohistiocytosis in children. J Pediatr. 2012;160(6):984-990.e1.

28. Yang SL, Xu XJ, Tang YM, et al. Associations between inflammatory cytokines and organ damage in pediatric patients with hemophagocytic lymphohistiocytosis. Cytokine. 2016;85:14-17.

29. Jordan MB, Hildeman D, Kappler J, Marrack P. An animal model of hemophagocytic lymphohistiocytosis (HLH): CD8+ T cells and interferon $\gamma$ are essential for the disorder. Blood. 2004;104(3):735-743.

30. Paczesny S. Biomarkers for posttransplantation outcomes. Blood. 2018;131(20):21932204

31. Paczesny S, Hakim FT, Pidala J, et al National Institutes of Health Consensus Development Project on Criteria for Clinical Trials in Chronic Graft-versus-Host Disease: III. The 2014 Biomarker Working Group Report. Biol Blood Marrow Transplant. 2015;21(5):780-792

32. Groom JR, Luster AD. CXCR3 ligands: redundant, collaborative and antagonistic functions. Immunol Cell Biol. 2011;89(2): 207-215.
33. Fahmy NM, Yamani MH, Starling RC, et al Chemokine and chemokine receptor gene expression indicates acute rejection of human cardiac transplants. Transplantation. 2003;75(1):72-78.

34. Gupta A, Broin PO, Bao Y, et al. Clinical and molecular significance of microvascular inflammation in transplant kidney biopsies. Kidney Int. 2016;89(1):217-225.

35. Medoff BD, Wain JC, Seung E, et al. CXCR3 and its ligands in a murine model of obliterative bronchiolitis: regulation and function. Immunol. 2006;176(11):7087-7095.

36. An Q, Hu SY, Xuan CM, Jin MW, Ji Q, Wang $Y$. Interferon $\gamma$ and interleukin 10 polymorphisms in Chinese children with hemophagocytic lymphohistiocytosis. Pediatr Blood Cancer. 2017;64(9).

37. Locatelli F, Jordan M, Allen C, et al. Safety and efficacy of emapalumab in pediatric patients with primary hemophagocytic lymphohistiocytosis. Blood. 2018;132(Suppl 1):LBA-6.

38. Prencipe G, Caiello I, Pascarella A, et al Neutralization of interferon- $\gamma$ reverts clinical and laboratory features in a mouse model of macrophage activation syndrome. J Allergy Clin Immunol. $2018 ; 141(4): 1439-1449$.

39. Imahashi $N$, Inamoto $Y$, Ito $M$, et al. Clinical significance of hemophagocytosis in BM clot sections during the peri-engraftment period following allogeneic hematopoietic SCT. Bone Marrow Transplant. 2012; 47(3):387-394

40. Ferris RL, Lu B, Kane LP. Too much of a good thing? Tim-3 and TCR signaling in T cell exhaustion. J Immunol. 2014;193(4):15251530

41. Jin HT, Anderson AC, Tan WG, et al. Cooperation of Tim-3 and PD-1 in CD8 T cell exhaustion during chronic viral infection. Proc Natl Acad Sci U S A. 2010; 107(33):14733-14738

42. Ikehara Y, Yasunami Y, Kodama S, et al CD4(+) Valpha14 natural killer T cells are essential for acceptance of rat islet xenografts in mice. J Clin Invest. 2000; 105(12):1761-1767.

43. Lin $\mathrm{H}$, Nieda M, Rozenkov V, Nicol AJ Analysis of the effect of different NKT cell subpopulations on the activation of $\mathrm{CD} 4$ and CD8 T cells, NK cells, and B cells. Exp Hematol. 2006;34(3):289-295.

44. Zeglen S, Zakliczynski M, Wozniak-Grygiel $\mathrm{E}$, et al. Mixed cellular and humoral acute rejection in elective biopsies from heart transplant recipients. Transplant Proc. 2009; 41(8):3202-3205

45. Grant CR, Holder BS, Liberal R, et al Immunosuppressive drugs affect interferon (IFN)- $\gamma$ and programmed cell death 1 (PD-1) kinetics in patients with newly diagnosed autoimmune hepatitis. Clin Exp Immunol. 2017;189(1):71-82. 